\title{
MODELLING LIVER TISSUE PROPERTIES USING A NON-LINEAR VISCOELASTIC MODEL FOR SURGERY SIMULATION*
}

\author{
Jean-Marc Schwartz $^{1}$, Marc Dellinger ${ }^{2}$, Denis Rancourt $^{2}$, Christian Moisan $^{3}$ \\ AND DENIS LAURENDEAU ${ }^{1}$
}

\begin{abstract}
We introduce an extension of the linear elastic tensor-mass method which allows fast computation of non-linear and viscoelastic mechanical deformations, and is suitable for the simulation of biological soft tissue deformation. We aim at developing a simulation tool for the planning of cryogenic surgical treatment of liver cancer. Percutaneous surgery simulation requires accurate modeling of the mechanical behavior of soft tissues, and experimental characterizations have shown that linear elasticity is only a coarse approximation of the real properties of biological tissues. We first show that our model can simulate different types of non-linear and viscoelastic mechanical behaviors at speeds which are compatible with real-time applications. Then an experimental setup is presented which was used to characterize the mechanical properties of deer liver tissue under perforation by a biopsy needle. Experimental results demonstrate that a linear model is not suitable for simulating this application while the proposed model succeeds in accurately modeling the mechanical behavior of liver tissue.
\end{abstract}

Résumé. Nous présentons une extension de la méthode des masses-tenseurs élastique linéaire permettant le calcul de déformations mécaniques non-linéaires et viscoélastiques, et adaptée à la simulation de déformations de tissus biologiques mous. Notre objectif est de développer un outil de simulation du traitement du cancer du foie par cryochirurgie. La simulation de chirurgie percutanée nécessite une modélisation précise du comportement mécanique des tissus mous, et plusieurs caractérisations expérimentales ont montré que le modèle élastique linéaire n'était qu'une approximation imprécise des propriétés de tissus biologiques. Nous montrons d'abord que notre modèle peut simuler différents types de comportements mécaniques non-linéaires et viscoélastiques à des vitesses compatibles avec la construction d'applications en temps réel. Puis nous présentons un montage expérimental utilisé pour caractériser les propriétés mécaniques du foie de cerf lors de la perforation par une aiguille à biopsie. Les résultats expérimentaux démontrent qu'un modèle linéaire n'est pas adapté à la simulation d'une telle application, alors que le modèle proposé est capable de reproduire avec précision le comportement mécanique du foie.

\section{INTRODUCTION}

The development of surgery simulation systems requires fast algorithms to allow real-time computation of tissue deformations, as well as accurate modeling of soft tissue mechanical behavior. We are currently developing a simulation tool for the planning of percutaneous image-guided cryosurgical treatment of liver

* This work was supported by NSERC Grant no. 234773 under research project SKALPEL.

1 Computer Vision and Systems Laboratory, Laval University, Québec (Qc) G1K 7P4, Canada

2 Biomechanics Laboratory, Laval University, Québec (Qc) G1K 7P4, Canada

3 iMRI Unit, Quebec City University Hospital, Québec (Qc) G1L 3L5, Canada 
cancer. This therapy consists in destroying tumor cells through successive application of freezing and passive thawing cycles [9]. Careful planning is required to optimize the destruction of tumor cells while limiting damage to surrounding healthy cells. For the simulation system to be efficient, accurate modeling of the geometric, thermal, and mechanical behavior of organs is required.

Several methods have been reported for fast calculation of linear elastic mechanical deformations. Some of them are based on non-physical constructions, such as the linked volume representation introduced by Gibson et al. [4]. Physical models are more widely used, from relatively simple models such as the spring-mass model [5] to models based on continuum mechanics such as the Finite Element Method [3]. However, experimental characterizations suggest that linear elasticity is only a coarse approximation of the real properties of biological soft tissues. For example Miller et al. [8] identified a viscoelastic constitutive model as accurate for modeling brain tissue deformations. Pioletti et al. [11] studied the properties of human knee ligaments and introduced a model containing a linear elastic term and two viscoelastic terms.

Since surgery simulation requires accurate description of soft tissues, we moved towards a more precise mechanical model than linear elasticity. The most promising approach towards real-time computation of nonlinear viscoelasticity appeared to be the tensor-mass model introduced by Cotin et al. [3]. The tensor-mass algorithm for linear elasticity is both time-efficient and physically accurate. It also allows local topological changes on mesh elements so that simulation of cutting or perforation is possible.

\section{Mechanical Model}

\subsection{Tensor-mass system}

The dynamic linear elastic tensor-mass model was introduced by Cotin et al. [3]. The modeled object is discretized into a conformal tetrahedral mesh as defined by finite element theory. Inside every tetrahedron $T_{i}$, the displacement field is defined by a linear interpolation of the displacement vectors of the four vertices of the tetrahedron. The linear elastic energy of tetrahedron $T_{i}$ can then be expressed as a function of the displacements of the four vertices and of the two Lamé coefficients of the material $\lambda_{i}$ and $\mu_{i}$. The force $\mathbf{F}_{T_{i}(j)}$ applied to a summit $\mathbf{P}_{T_{i}(j)}$ of tetrahedron $T_{i}$ is defined as the derivate of the elastic energy and takes the following form:

$$
\mathbf{F}_{T_{i}(j)}=\sum_{k=0}^{3}\left[\mathbf{K}_{j k}^{T_{i}}\right] \cdot \mathbf{P}_{T_{i}(k)}^{0} \mathbf{P}_{T_{i}(k)}
$$

where $\mathbf{P}_{T_{i}(k)}^{0}$ are the rest positions of the four vertices of tetrahedron $T_{i}, \mathbf{P}_{T_{i}(k)}$ are the current positions of the vertices, and $\left[\mathbf{K}_{j k}^{T_{i}}\right]$ are $3 \times 3$ stiffness tensors depending only on the rest geometry of tetrahedron $T_{i}$ and on the Lamé coefficients. These tensors can be precomputed, therefore computation at run-time is restricted to matrix-vector multiplications and matrix summations.

Given a complete mesh, the total elastic force $\mathbf{F}_{i}$ applied on a vertex $\mathbf{P}_{i}$ is obtained by summing the forces contributed by all adjacent tetrahedra of $T_{i}$ :

$$
\mathbf{F}_{i}=\left[\mathbf{K}_{i i}\right] \cdot \mathbf{P}_{i}^{0} \mathbf{P}_{i}+\sum_{j \in N\left(P_{i}\right)}\left[\mathbf{K}_{i j}\right] \cdot \mathbf{P}_{j}^{0} \mathbf{P}_{j}
$$

where $\left[\mathbf{K}_{i i}\right]$ is the sum of tensors $\left[\mathbf{K}_{i i}^{T_{k}}\right]$ associated with the tetrahedra adjacent to $\mathbf{P}_{i},\left[\mathbf{K}_{i j}\right]$ is the sum of tensors $\left[\mathbf{K}_{i j}^{T_{k}}\right]$ associated with the tetrahedra adjacent to edge $(i, j)$, and $N\left(\mathbf{P}_{i}\right)$ is the neighborhood of vertex $\mathbf{P}_{i}$.

The resulting system has to be solved dynamically. The motion of the system is determined by a Newtonian equation which takes the form:

$$
m_{i} \ddot{\mathbf{P}}_{i}+\gamma_{i} \dot{\mathbf{P}}_{i}-\mathbf{F}_{i}=0
$$

where $m_{i}$ is the mass associated to vertex $\mathbf{P}_{i}$ and $\gamma_{i}$ the damping coefficient associated to vertex $\mathbf{P}_{i}$. In relation (3) the mass and damping effects are supposed to be lumped at vertices. 
Different integration schemes can be chosen to solve the dynamic system [1]. We chose an explicit scheme based on the Euler method, because it offers the best compromise between computational speed and stability of the system. Vertex position at time $t+h$ is derived from its positions at times $t$ and $t-h$, and from the elastic force computed at time $t$ :

$$
\mathbf{P}_{i}(t+h)=\frac{1}{m_{i}+\gamma_{i} h}\left(h^{2} \mathbf{F}_{i}(t)+\left(2 m_{i}+\gamma_{i} h\right) \mathbf{P}_{i}(t)-m_{i} \mathbf{P}_{i}(t-h)\right)
$$

\subsection{Non-linearity modeling}

In equation (1), the force field is expressed as a linear combination of the displacements of the tetrahedron vertices. One way to extend the model to non-linearity consists in considering higher order terms in the expression of elastic energy. For example Picinbono et al. [10] describe a non-linear tensor-mass model based on the St Venant-Kirchhoff elastic model which adds quadratic and cubic terms to expression (1). We rather chose to keep a linear expression for the force and introduce non-linearity by acting on the stiffness tensor itself so as to minimize additional computational burden. Modification of the stiffness tensor cannot be done in any possible way but has to satisfy the isotropy principle for the material. Only two degrees of freedom remain when all space symmetries have been considered, and they correspond to the two Lamé coefficients [2]. Therefore acting on the Lamé coefficients is an easy way to modify the elastic properties of the material in real-time while satisfying all isotropy constraints.

The expression of tensor $\left[\mathbf{K}_{j k}^{T_{i}}\right]$ can be divided into two components proportional to $\lambda_{i}$ and $\mu_{i}$ respectively:

$$
\left[\mathbf{K}_{j k}^{T_{i}}\right]=\lambda_{i}\left[\mathbf{A}_{j k}^{T_{i}}\right]+\mu_{i}\left[\mathbf{B}_{j k}^{T_{i}}\right]
$$

where $\left[\mathbf{A}_{j k}^{T_{i}}\right]$ and $\left[\mathbf{B}_{j k}^{T_{i}}\right]$ also are $3 \times 3$ tensors depending only on the rest geometry of tetrahedron $T_{i}$, which can be precomputed. Therefore a non-linear expression can be computed under the form:

$$
\mathbf{F}_{T_{i}(j)}=\sum_{k=0}^{3}\left(\left[\mathbf{K}_{j k}^{T_{i}}\right]+\delta \lambda\left(T_{i}\right)\left[\mathbf{A}_{j k}^{T_{i}}\right]+\delta \mu\left(T_{i}\right)\left[\mathbf{B}_{j k}^{T_{i}}\right]\right) \cdot \mathbf{P}_{T_{i}(k)}^{0} \mathbf{P}_{T_{i}(k)}
$$

where $\delta \lambda\left(T_{i}\right)$ and $\delta \mu\left(T_{i}\right)$ are the non-linear corrections. $\delta \lambda$ and $\delta \mu$ depend on the intensity of deformation of the tetrahedron. The choice in their expression as a function of the shape of the tetrahedron determines the kind of non-linear law being simulated. Examples of possible simulated laws are shown in section 2.2.

A quantization of the tetrahedron deformation intensity has to be defined as argument for $\delta \lambda$ and $\delta \mu$. Several tetrahedron shape measures exist and are commonly used for assessing the quality of tetrahedra in finite element meshes. Liu and Joe [7] discussed several of these measures and showed that they are equivalent, in the sense that if one measure approaches zero, so do the others, and that all measures attain a maximum value only for the regular tetrahedron. From that property, we assume that the choice of a particular shape measure does not significantly affect the overall behavior of the system, and that this choice should be mainly directed towards computational efficiency. With this in mind we chose to use the tetrahedron mean ratio as defined by Liu and Joe $[6]$.

\subsection{Viscoelastic modeling}

Experimental characterizations reveal that viscous effects cannot be ignored for an accurate description of the mechanical properties of biological tissues. Viscoelasticity can easily be introduced into the tensor-mass model, provided that the behavior be restricted to a simple linear viscous relation. We introduced a viscous force that is proportional to the speed of deformation and to a viscosity coefficient $\eta_{i}$. After discretization onto a tetrahedral mesh, the expression obtained is very similar to (1), except that deformation speed replaces deformation, and a viscosity tensor replaces the stiffness tensor. Expression of the viscous force $\mathbf{F}_{T_{i}(j)}^{(v)}$ applied 


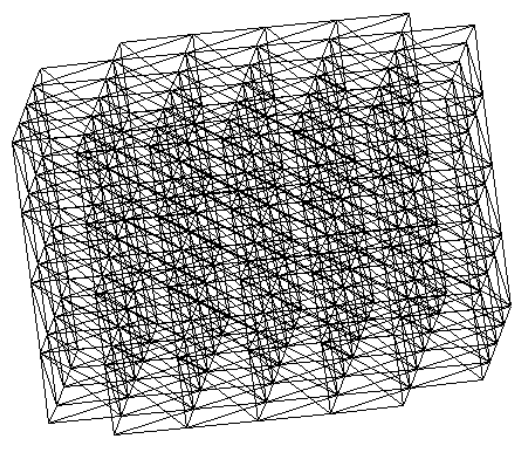

a)

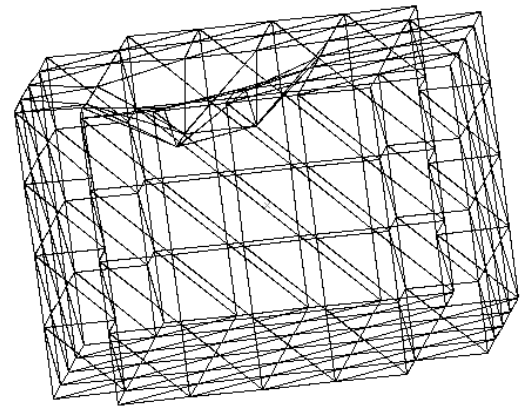

b)

Figure 1. a) Undeformed mesh. b) Deformed mesh after compression was applied onto the top. Only external edges are shown in b) for better clarity

to a vertex $\mathbf{P}_{T_{i}(j)}$ of tetrahedron $T_{i}$ is then:

$$
\mathbf{F}_{T_{i}(j)}^{(v)}=\sum_{k=0}^{3}\left[\mathbf{K}_{j k}^{T_{i}}\right]^{(v)} \cdot \frac{d}{d t} \mathbf{P}_{T_{i}(k)}^{0} \mathbf{P}_{T_{i}(k)}
$$

where $\left[\mathbf{K}_{j k}^{T_{i}}\right]^{(v)}$ are $3 \times 3$ tensors depending only on the rest geometry of tetrahedron $T_{i}$ and on the viscosity coefficient $\eta_{i}$. The effect of the viscous term on the simulated mechanical behavior is illustrated in section 2.3 .

\section{Evaluation of THE MECHANiCAL MODEL}

\subsection{Model mesh}

We performed a series of simulations in order to check the ability of our model to reproduce different types of mechanical behavior. The mesh used for these tests is shown in figure 1a. It is a regular mesh composed of 225 vertices and 768 tetrahedra. The length of the edge of each cubic compound is $1 \mathrm{~cm}$, and each cubic compound is divided into 6 tetrahedra. Compression is applied onto a triangular surface on top of the mesh. Figure 1b shows an example of deformed configuration after compression.

\subsection{Non-linearity simulation}

Figure 2 shows examples of simulated non-linear tissue constitutive laws. Figure 2a plots defined relations between Young's modulus $E$ and the tetrahedron mean ratio, and Figure 2b plots the resulting force obtained in simulated compression. For tissue 1, stiffness increases sharply at a given ratio value. This results in the force growing stepwise during compression, a new step is added every time a new mesh element reaches the threshold value. For tissue 2, stiffness increases linearly with the ratio value. This results in the force being close to a second order curve. In both cases, simulation on a macroscopic mesh correctly follows the constitutive law that was defined.

\subsection{Viscoelasticity simulation}

The effect of the viscous part of our tissue model is analyzed in Figure 3. In figure 3a, a tissue with viscosity coefficient $\eta=1000 \mathrm{~Pa} \cdot \mathrm{s}$ was modeled, and compression was applied with different velocities. In figure $3 \mathrm{~b}$, tissues with different viscosity coefficients were modeled and compressed with the same velocity of $10 \mathrm{~mm} / \mathrm{s}$. 


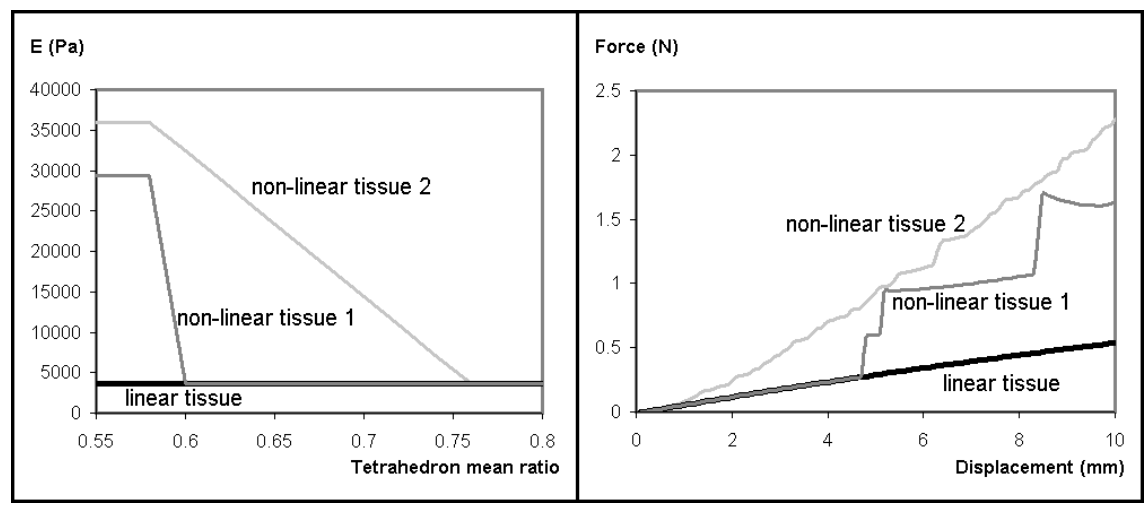

a)

b)

Figure 2. a) Relation between Young's modulus $E$ and the tetrahedron mean ratio for three simulated tissues. For the linear case $E=3600 \mathrm{~Pa}$. For all three tissues the Poisson coefficient was $\nu=0.4$. b) Resulting force as a function of displacement in a simulated compression experiment. Compression velocity was $10 \mathrm{~mm} / \mathrm{s}$, time step for each iteration was $0.01 \mathrm{~s}$

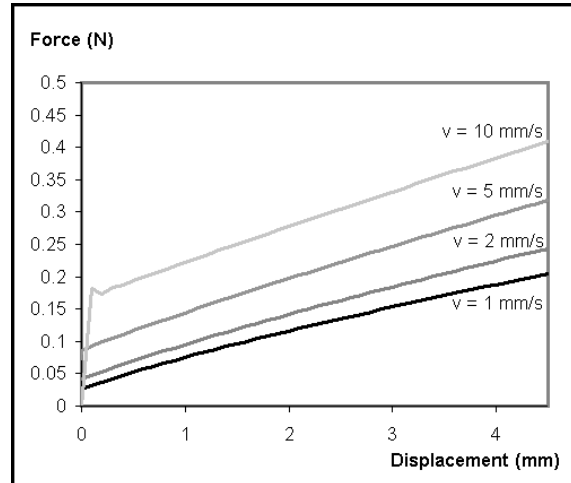

a)

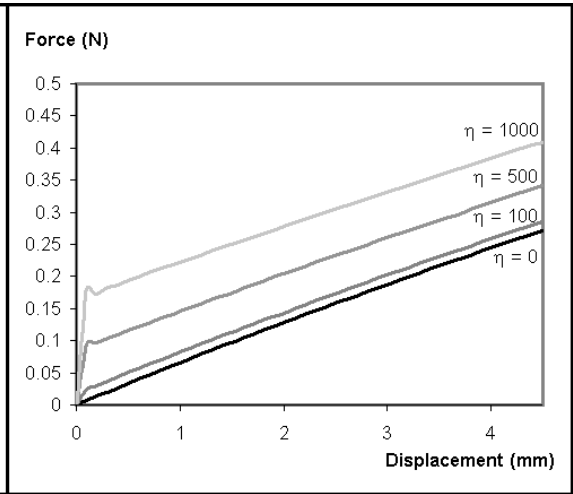

b)

FIGURE 3. Force as a function of displacement for simulated viscoelastic tissues. For all curves, no non-linear function was introduced into the tissue model and compression is applied with constant velocity. a) Different compression velocities for the same tissue of viscosity coefficient $\eta=1000 \mathrm{~Pa} \cdot \mathrm{s}$. b) Compression is applied with a velocity of $10 \mathrm{~mm} / \mathrm{s}$ onto tissues with different viscosity coefficients

The effect of the viscous term is an increased tissue resistance at higher compression speeds, as could be expected. Because velocity was constant from the start in these simulations, a force overhead appears as soon as compression starts. It can be noted that because of the dynamical nature of the model, a tissue model with no viscosity still exhibits limited viscous behavior, since the force field propagates more slowly through the mesh at higher compression speeds. This can be seen by comparing the curve for $\eta=1000, v=1$ on figure 3 a with the curve for $\eta=0, v=10$ on figure $3 \mathrm{~b}$.

\subsection{Computation speed}

On a $550 \mathrm{MHz}$ Pentium II computer with $512 \mathrm{MB}$ RAM, processing 100 iterations using the complete viscoelastic model on the 768 tetrahedra mesh shown in figure 1 took $6.5 \mathrm{~s}$. The complete viscoelastic model 

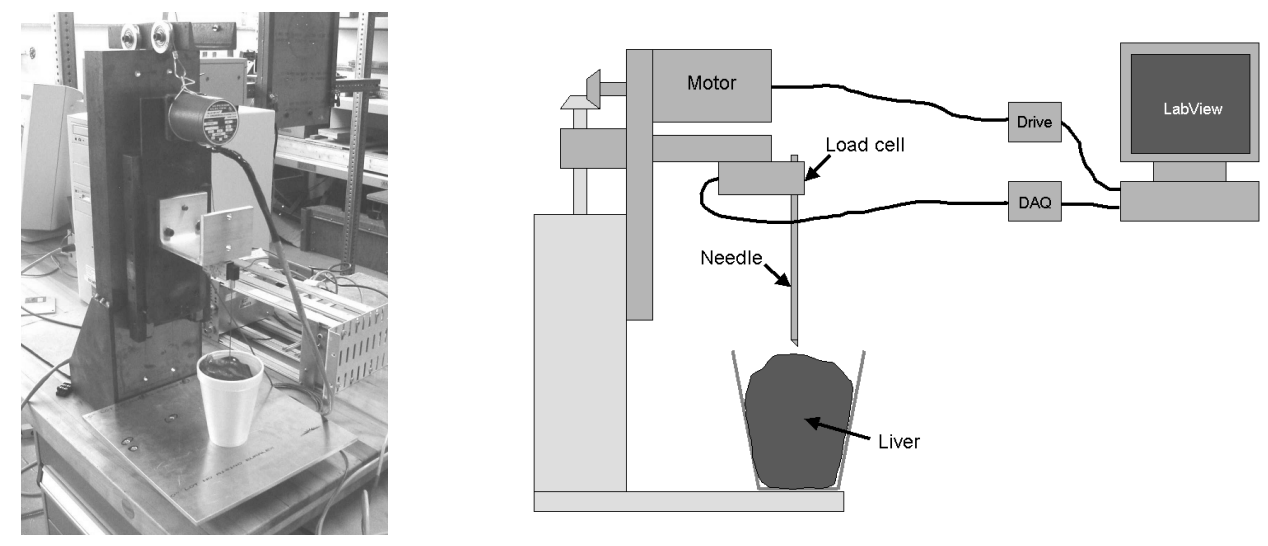

Figure 4. Experimental setup for the characterization of mechanical properties of liver

takes 5 times more computation time than the linear elastic tensor-mass method. The method remains suitable to real-time applications, as several improvements can been considered in order to improve performance. The non-linear and viscous computational overheads may be focused to a limited number of mesh elements where the highest deformation rates occur. Parallel computing may be considered as well by dividing the complete mesh into several submeshes.

\section{EXPERIMENTAL VALIDATION}

\subsection{Experimental setup}

We showed that the extended tensor-mass method presented above allows to compute non-linear viscoelastic deformations at rates compatible with real-time applications. The next goal was to check whether this model can accurately simulate the mechanical behavior of real biological soft tissue.

For this purpose, we designed an experimental setup to characterize the mechanical properties of biological tissue in a perforation experiment. Experimental characterization was necessary because there is very little data available in the literature about mechanical properties of very soft tissues such as liver. Furthermore precise data is needed to adjust the parameters of the simulation model for further clinical applications.

The experimental setup is shown in figure 4. A $24 \mathrm{~mm}$ diameter biopsy needle was mounted on a 5 lbs Totalcomp TMB-5 load cell. Vertical movement was controled by a step-motor whose velocity ranges from 0 to $10 \mathrm{~mm} / \mathrm{s}$. The needle perforated a sample of deer liver placed in a cylindrical container. The force exerted onto the needle and needle position were acquired at $500 \mathrm{~Hz}$ by an A/D sampling board and plotted.

\subsection{Results}

A series of measurement have been conducted on deer liver using this setup. The needle was moved vertically at constant velocity and compressed the liver until perforation of the liver membrane. The force exerted onto the load cell was measured and plotted against needle displacement. Three different velocities were considered, 2, 6 and $10 \mathrm{~mm} / \mathrm{s}$. For each velocity, 10 independent experiments were carried out.

Results showed to be highly reproducible. At a given velocity, force curves are very similar, independently of the position where the needle was applied. Only the point where membrane rupture occurs is variable, but this variability does not affect the behavior before rupture. We first used 5 curves taken at $2 \mathrm{~mm} / \mathrm{s}$ to estimate the parameters used in the simulation model and reproduced the experimental conditions in simulation. The black curve on figure 5 a plots the simulated force while the light gray curves are those used for parameter adjustment.

On figure $5 \mathrm{~b}$, the same simulated curve is plotted along with the 5 other experimental curves measured for a speed of $2 \mathrm{~mm} / \mathrm{s}$, which were not used to determine simulation parameters. They show very good correspondence 


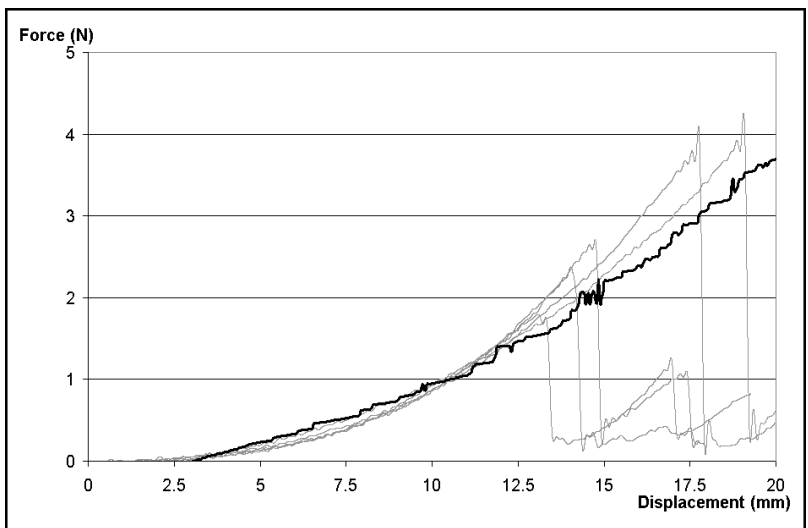

a)

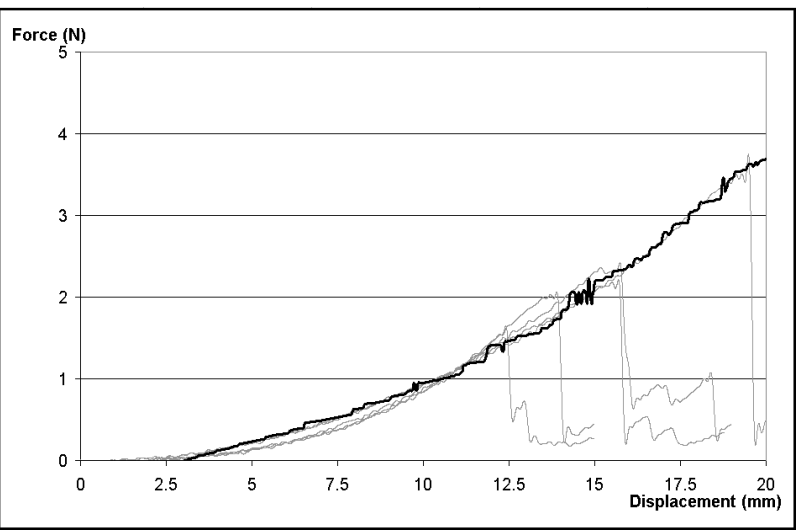

b)

FiguRE 5. Comparison between experimental and simulated force in liver tissue perforation. The needle was moved vertically with a constant velocity of $2 \mathrm{~mm} / \mathrm{s}$. Simulated curve is in black, experimental curves are in light gray. a) The 5 measurements used to adjust the parameters of the simulation model. b) 5 other independent measurements. Experimental curves were filtered for display clarity

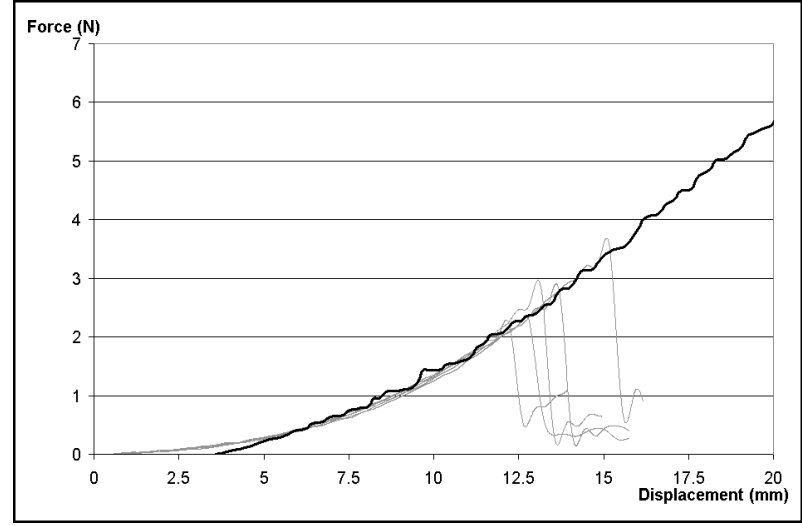

a)

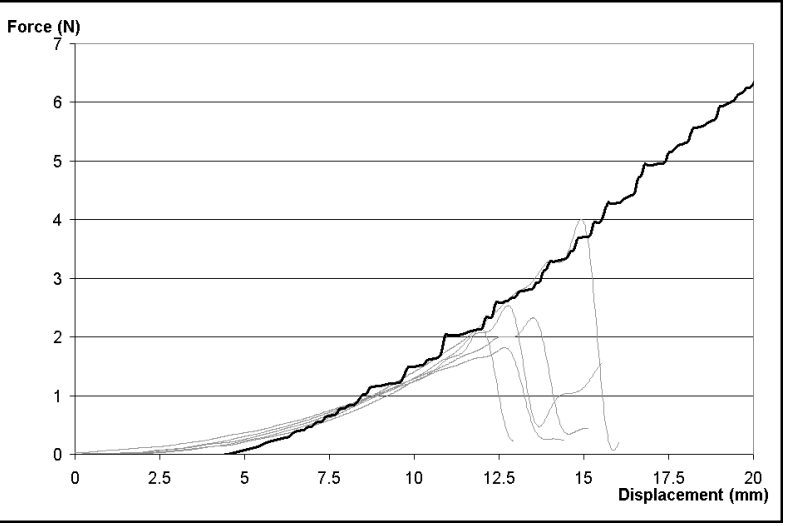

b)

Figure 6. Comparison between experimental and simulated force for different perforation velocities. Simulated curve is in black, experimental curves are in light gray. a) Velocity is 6 $\mathrm{mm} / \mathrm{s}$. b) Velocity is $10 \mathrm{~mm} / \mathrm{s}$. Experimental curves were filtered for display clarity

to the simulation curve as well. Only the behavior before rupture of the liver membrane has been modeled; the sharp drop of the experimental curves corresponds to membrane rupture.

Finally the parameters derived from measurements at $2 \mathrm{~mm} / \mathrm{s}$ were used as such to simulate perforations at other velocities. Figure 6 a displays results for a speed of $6 \mathrm{~mm} / \mathrm{s}$, figure $6 \mathrm{~b}$ for $10 \mathrm{~mm} / \mathrm{s}$. The black curve shows simulation, and the light gray curves show 5 independent measurements. For both velocities, the model closely fits experimental data.

No systematic method has been used to fit model parameters, so different combinations of parameters may be able to give equivalent or better results. An algorithm remains to be designed to conduct the fitting process in order to ensure that the optimal solution would be found. 


\section{Conclusion}

We introduced an extension of the linear elastic tensor-mass method which allows fast computation of nonlinear viscoelastic deformations and is suitable for the simulation of biological soft tissue deformation. Several experimental characterizations allowed us to demonstrate that this model succeeds in reproducing the mechanical behavior of liver tissue in a compression experiment. To reach the goal of complete simulation of soft tissue perforation, the behavior after perforation of the tissue membrane remains yet to be modeled. For a more global characterization of the mechanical behavior, additional force or deformation measurement data would be needed. A more complete experimental setup is under planning which will include load measurements on the bottom and the sides of the tissue container. Additionally, performance of the method can be further improved by the use of parallel computing and by a dynamical selection of the precision of the model depending on local deformation conditions.

\section{REFERENCES}

[1] Bro-Nielsen, M.: Finite Element Modeling in Surgery Simulation. Proc. of the IEEE 86 (1998) $490-503$.

[2] Chou, P. C., and Pagano, N. J.: Elasticity: Tensor, Dyadic, and Engineering Approaches. Van Nostrand Inc. (1967).

[3] Cotin, S., Delingette, H., and Ayache, N.: A Hybrid Elastic Model for Real-Time Cutting, Deformations, and Force Feedback for Surgery Training and Simulation. Visual Computer 16 (2000) 437-452.

[4] Gibson, S. F. F.: Using Linked Volumes to Model Object Collisions, Deformation, Cutting, Carving, and Joining. IEEE Transactions on Visualization and Computer Graphics 5 (1999) 333-348.

[5] Kühnapfel, U., and Neisius, B.: CAD-based Graphical Simulation in Endoscopic Surgery. Endoscopic Surgery and Allied Technologies 1 (1993) 181-184.

[6] Liu, A., and Joe, B.: On the Shape of Tetrahedra from Bisection. Mathematics of Computation 63 (1994), $141-154$.

[7] Liu, A., and Joe, B.: Relationship between Tetrahedron Shape Measures. BIT 34 (1994) $268-287$.

[8] Miller, K., Chinzei, K., Orssengo, G., and Bednarz, P.: Mechanical Properties of Brain Tissue in-vivo: Experiment and Computer Simulation. J. Biomech. 33 (2000) 1369-1376.

[9] Morin, J., Dionne, G., Dumont, M., Fouquet, B., Dufour, M., Cloutier, S., and Moisan, C.: MR Guided Percutaneous Cryosurgery of Breast Carcinoma: Technique and Early Clinical Results. Proc. Int. Soc. Mag. Res. in Med. (2000) 71.

[10] Picinbono, J., Delingette, H., and Ayache, N.: Non-linear and Anisotropic Elastic Soft Tissue Models for Medical Simulation. Proc. ICRA 2001 (International Conference on Robotics and Automation, Seoul, Korea, May 2001) 1370-5.

[11] Pioletti, D. P., Rakotomanana, L. R., Benvenuti, J.-F., and Leyvraz, P.-F.: Viscoelastic Constitutive Law in Large Deformations: Application to Human Knee Ligaments and Tendons. J. Biomech. 31 (1998) 753-757. 